\title{
Radix Cyathula officinalis Kuan inhibits arterial remodeling in spontaneously hypertensive rats
}

\author{
JIAJING ZHAO*, YAOHAN YUE* , YUN XIE, LIWEN LIU, FEI CAO, \\ SHURONG GAO and YINGJUE WANG
}

\begin{abstract}
Department of Traditional Chinese Medicine, Shanghai Putuo People's Hospital, Shanghai 200060, P.R. China
\end{abstract}
Received May 24, 2017; Accepted September 5, 2017

DOI: 10.3892/etm.2017.5218

\begin{abstract}
There is still no resolution for arterial remodeling related with hypertension, though hypertension treatment has access to a number of pharmacological agents. The present study aimed at investigating the prevention of Cyathula officinalis Kuan's roots (C. officinalis Kuan) against in arterial remodeling in vitro. Spontaneously hypertensive rats (SHRs) were intragastrically administered 3,6 or $12 \mathrm{~g} / \mathrm{kg}$ C. officinalis Kuan or normal saline or enalapril $(2.5 \mathrm{mg} / \mathrm{kg})$ once a day for 8 weeks. Hematoxylin and eosin were used to measure blood pressure and stain carotid and arota. The serum concentration of nitric oxide (NO) was measured by NO assay kit (nitrate reductase method). The endothelin-1 transcriptional level, endothelial NO synthase of endothelium as well as angiotensin II receptor type 1 (AT1R) of aorta and carotid was tested by quantitative reverse transcriptionpolymerase chain reaction (qRT-PCR) and the protein level in aorta was also measured by western blotting. The blood pressure in SHR+enalapril, SHR+3 g/kg, SHR+6 g/kg and $\mathrm{SHR}+12 \mathrm{~g} / \mathrm{kg}$ C. officinalis Kuan groups was significantly decreased at 4, 6 and 8 weeks post-treatment compared with SHR group. Different doses of $C$. officinalis Kuan and enalapril treatment showed aortic wall thinness and strengthened NO serum level, but made no impact on the transcriptional level of AT1R in aorta or endothelial NO synthase in carotid. It is suggested by such results that therapy by $C$. officinalis Kuan is able to fight against arterial remodeling, thus may provide a new means to treat arterial remodeling caused by hypertension.
\end{abstract}

Correspondence to: Dr Jiajing Zhao or Dr Yingjue Wang, Department of Traditional Chinese Medicine, Shanghai Putuo People's Hospital, 1291 Jiangning Road, Putuo, Shanghai 200060, P.R. China E-mail: zhaojiajingrose@126.com

E-mail: wangyjsh@126.com

${ }^{*}$ Contributed equally

Key words: Radix Cyathula officinalis Kuan, hypertension, arterial remodeling, ET-1, eNOS, AT1R

\section{Introduction}

The contribution of hypertension to mortality and morbidity in people's health is preventable, for its etiologic influence and its growing influence in stroke, kidney failure and heart attack (1). Based on the reports from World Health Organization (WHO), high levels of blood pressure, even when just suboptimal, have responsibility for $49 \%$ ischaemic heart disease and $62 \%$ cerebrovascular disease (2). The rise of BP within a patient with hypertension results from control mechanisms for blood pressure, such as vascular resistance of periphery, volume of circulating blood and cardiac output. It is a crucial task to choose the appropriate therapy for every patient (3).

During the past years, invasive and non-invasive techniques have brought improved vascular changes within experimental animals and hypertensive patients (4). In both animal and human models, there is an association between hypertension and aortic remodeling (5), which is featured by structural vascular alterations and destroyed endothelium-dependent vasodilation (6). Therefore, endothelium is essential to the vascular structure and tone (7). A declined aortic diameter within hypertensive subject in middle age may also make sense to increase pulse pressure via strengthening particular impedance, which contradicts the traditional phenotype of hypertensive aortic featured by degenerated and calcific vascular wall and increased aortic diameter (8).

To account for various mechanisms of blood pressure, scientists have developed the therapy of targeted anti-hypertension. Even though anti-hypertensive drugs, like calcium-channel blockers, receptor blockers of angiotensin II (Ang II) and inhibitors of angiotensin-converting enzyme (ACE), have extreme application in clinical treatment, there is no resolution for vascular changes induced by hypertension (9). Thus, it is necessary to develop new therapeutic tactics and drugs for vascular remodeling related with hypertension. With the characteristics of 'multi-target', composition of many compatible herbs and multiple compounds in one prescription, conventional Chinese herbs have achieved a good acceptance in China that attempts to decrease side effects and promote efficacy (10). Cyathula officinalis (C. officinalis), with family of Amaranthaceae family, belongs to an herbaceous plant perennially with wide distribution in tropical regions of Africa and Asia, and especially 
in Korea, Vietnam and China. C. officinalis Kuan's roots, C. officinalis Kuan, in Chinese called 'Chuan Niu Xi', have functions to remove blood stasis and restore menstrual flow, ease joint movement, as well as induce diuresis for treatment of stranguria (11). It is often applied as emmenagogue, atonic, antiarthritic, anti-fertility agent and diuretic to nourish kidneys and liver, fortify muscles and bones, and activate circulation (12). C. officinalis Kuan has been extracted with diverse active compounds in biology, such as palmitic acids, hyterocyclic compounds and phytoecdysteroids $(13,14)$, whose biological attributes have been featured. Nevertheless, the antihypertensive attributes of $C$. officinalis Kuan have attracted little attention.

In the present study, we made efforts to evaluate the impacts of $C$. officinalis Kuan on the arterial remodeling in spontaneously hypertensive rats (SHRs). The results indicated that $C$. officinalis Kuan could improve the arterial remodeling by decreasing endothelin-1 (ET-1) and increasing endothelial nitric oxide synthase (eNOS) and ATIR expression.

\section{Materials and methods}

Animal treatments. Male rats, 12 -weeks-old with spontaneous hypertension (SHR) (245-285 g) were obtained from the Shaanxi Jiahe Phytochem Co., Ltd. (Xian, China). SHR were separated into 5 groups randomly with 8 rats in each group: SHR treated by $0.9 \%$ saline were considered to be a model of hypertension (SHR); SHR in the other 4 groups were administered with 3,6 and $12 \mathrm{~g} / \mathrm{kg} C$. officinalis Kuan or $2.5 \mathrm{mg} / \mathrm{kg}$ enalapril. The rats had a dark/light cycle of $12 / 12 \mathrm{~h}$ at fixed temperature of $22-23^{\circ} \mathrm{C}$ with available water and food freely. The administration was once a day for eight weeks. Monitoring of blood pressure was once a week using a tail BP Series Automatic non-invasive blood pressure measuring system (BP-300A; Chengdu Techman Software Co., Ltd., Chengdu, China) during the experimental period. Animal Care and Use Committee of Shanghai Putuo People's Hospital approved this study according to the guidelines on Ethical Care for Experimental Animals.

Histological assessment. At the end of the experiments, the rats were euthanized with an overdose of chloral hydrate. The aorta of the rats was harvested, and fixed with $10 \%$ formalin, dehydrated and embedded into paraffin. Next, sections with thickness of $4 \mu \mathrm{m}$ were cut, and then stained with hematoxylin and eosin (H\&E). The Olympus BX51 microscope with the camera of Olympus DP71 CCD from Olympus (Tokyo, Japan) was used to capture digital images (magnification, $\mathrm{x} 200$ ). A blinded manner was used to perform analysis on all images.

Measurement of serum NO level. The serum concentration of nitric oxide (NO) was measured by Nitric Oxide assay kit (Nitrate reductase method, A012; Nanjing Jiancheng Bioengineering Institute, Nanjing, China) in accordance with instructions of the manufacturer.

RNA extraction and analysis on quantitive reverse transcription-polymerase chain reaction ( $q R T-P C R)$. Whole RNA was extracted from aorta by snap-freezing and samples of carotid by RNAiso Plus and PrimeScript reagent kit of reverse reaction (DRR037A) (both from Takara, Dalian, China) was used to carry out reverse transcription reaction on RNA in accordance with manufacturer's instructions. Quantitative analysis on the change of expression level was conducted by SYBR Premix Ex Taq (DRR041A; Takara) in ABI 7500 (Thermo Fisher Scientific, Inc., Waltham, MA, USA). The primer sequences of PCR were: ET-1 forward, 5'-TGTTCCCT AACCTGTCTTC-3' and reverse, 5'-ACACTCCCTAAGGAC TTTC-3'; eNOS forward, 5'-CTTTCGGAAGGCGTTTGAC-3' and reverse, 5'-AACTCTTGTGCTGCTCAGG-3'; Ang II receptor type 1 (AT1R) forward, 5'-CTCTGTTCTACGGCT TTC-3' and reverse, 5'-CTTCTGTCAGGGCATTAC-3'; GAPDH forward, 5'-GTCGGTGTGAACGGATTTG-3' and reverse, 5'-TCCCATTCTCAGCCTTGAC-3'. The change in expression of mRNA within rats treated by saline, C. officinalis Kuan or enalapril was assessed by the $2^{-\Delta \Delta C q}$ method.

Western blotting. Whole protein was isolated out of snapfrozen aorta samples using radioimmunoprecipitation buffer, supplemented with protease inhibitor (Beyotime Institute of Biotechnology, Shanghai, China). The concentration of protein was estimated employing the assay kit of bicinchoninic acid (Thermo Fisher Scientific, Inc.). Equivalently quantitive protein $(30 \mu \mathrm{g})$ was divided subsequently on $12 \%$ SDS-PAGE gels, and then was moved onto membranes of nitrocellulose (EMD Millipore, Billerica, MA, USA). Following blocking, these membranes were immunoblotted overnight in $4^{\circ} \mathrm{C}$ with first antibodies: Anti-ET-1, anti-eNOS, anti-ATIR and anti-GAPDH. Horseradish peroxidase-conjugated second antibodies were used to incubate membranes after they were washed (1:1,000; Beyotime Institute of Biotechnology) for $1 \mathrm{~h}$ at $37^{\circ} \mathrm{C}$. Tris-buffered saline including Tween-20 of $20 \%$ was used to wash these membranes (Amresco, LLC, Solon, OH, USA). Detection for signals employed an improved system of chemiluminescence (Pierce, Rockford, IL, USA) and their determination employed software of ImageJ version 1.46 (National Institutes of Health, Bethesda, MD, USA).

Statistical analysis. The quantitive values are in mean \pm SD. GraphPad Prism software, version 5.0 (GraphPad Software, Inc., San Diego, CA, USA) was used to analyze nonlinear regression of each curve for dose-response. Calculation of data used one-way analysis on variance (ANOVA) and analysis on statistical calculations used SPSS 18.0 statistical software (SPSS Inc., Chicago, IL, USA). Comparison among data from various groups used one-way ANOVA. $\mathrm{P}<0.05$ was considered to indicate a statistically significant difference.

\section{Results}

C. officinalis Kuan therapy reduces blood pressure in SHR. Monitoring of blood pressure was conducted fortnightly at indicative time. In week 0 , different doses of $C$. officinalis Kuan $(3,6$ and $12 \mathrm{~g} / \mathrm{kg}$ ) or $2.5 \mathrm{mg} / \mathrm{kg}$ enalapril therapy did not show any impact on blood pressure in comparison to group of SHR (Table I). After 2 week, the blood pressure was $161.34 \pm 4.38,181.50 \pm 3.15,181.08 \pm 5.43$ and 
Table I. The blood pressure in SHR with enalapril or C. officinalis Kuan treatment.

\begin{tabular}{|c|c|c|c|c|c|}
\hline Groups & 0 week & 2 weeks & 4 weeks & 6 weeks & 8 weeks \\
\hline SHR & $185.67 \pm 6.31$ & $187.91 \pm 4.89$ & $191.48 \pm 6.51$ & $193.88 \pm 3.31$ & $195.51 \pm 4.88$ \\
\hline SHR+enalapril & $189.64 \pm 5.83$ & $161.34 \pm 4.38^{b}$ & $154.84 \pm 4.17^{b}$ & $155.31 \pm 4.38^{\mathrm{b}}$ & $154.29 \pm 4.38^{\mathrm{b}}$ \\
\hline $\mathrm{SHR}+3 \mathrm{~g} / \mathrm{kg}$ & $182.85 \pm 4.95$ & $181.50 \pm 3.15^{\mathrm{a}}$ & $181.74 \pm 3.21^{b}$ & $178.28 \pm 3.17^{b}$ & $177.63 \pm 5.49^{b}$ \\
\hline $\mathrm{SHR}+6 \mathrm{~g} / \mathrm{kg}$ & $186.81 \pm 3.74$ & $181.08 \pm 5.43^{\mathrm{a}}$ & $175.38 \pm 4.29^{b}$ & $172.00 \pm 4.11^{\mathrm{b}}$ & $167.21 \pm 6.11^{b}$ \\
\hline $\mathrm{SHR}+12 \mathrm{~g} / \mathrm{kg}$ & $181.34 \pm 5.84$ & $176.46 \pm 4.11^{\mathrm{b}}$ & $170.93 \pm 7.17^{\mathrm{b}}$ & $162.15 \pm 4.46^{\mathrm{b}}$ & $158.50 \pm 3.94^{b}$ \\
\hline
\end{tabular}

${ }^{\mathrm{a}} \mathrm{P}<0.05 ;{ }^{\text {b }}<0.01$ vs. SHR.

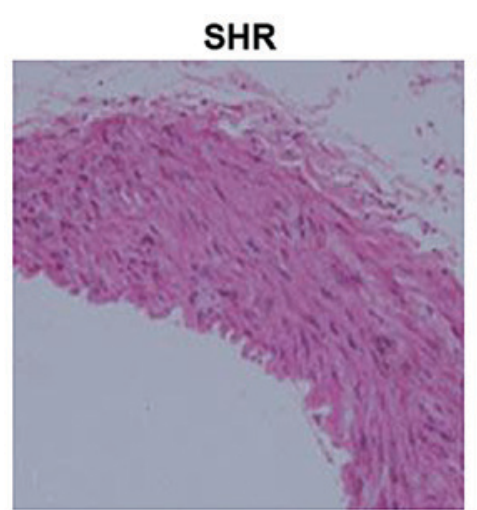

SHR+6 g/kg

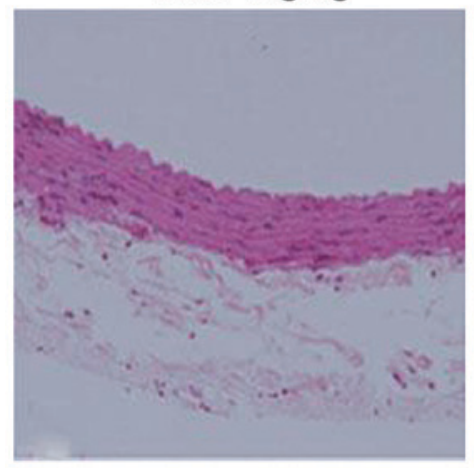

SHR+enalapril

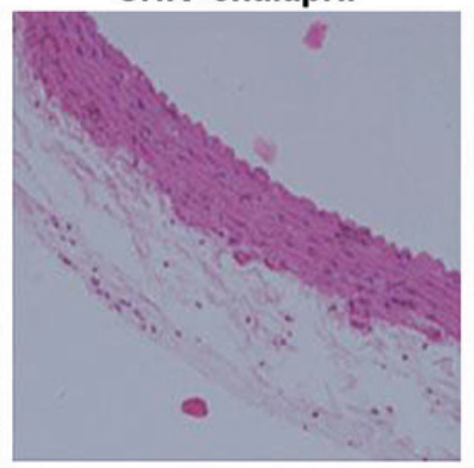

SHR+12 g/kg

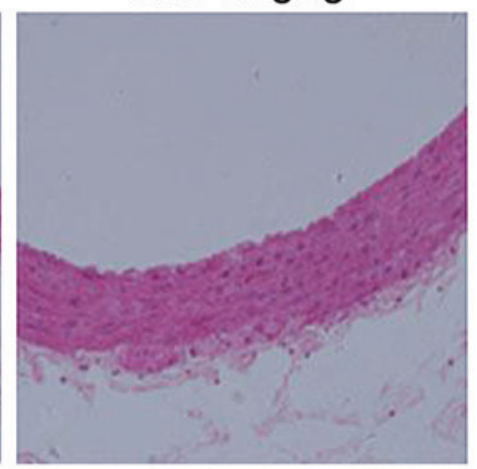

$\mathrm{SHR}+3 \mathrm{~g} / \mathrm{kg}$

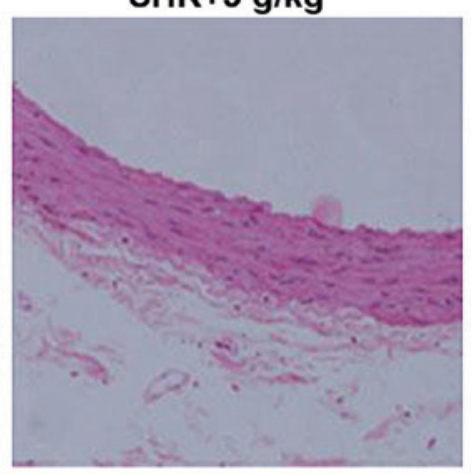

Figure 1. C. officinalis Kuan inhibits the thickness of aorta in SHR. After treatment of SHR with enalapril or different doses of $C$. officinalis Kuan (3, 6 or $12 \mathrm{~g} / \mathrm{kg}$ ), the arterial remodeling was evaluated using hematoxylin and eosin staining. C. officinalis, Cyathula officinalis; SHR, spontaneously hypertensive rat.

$176.46 \pm 4.11 \mathrm{mmHg}$ for enalapril, 3,6 and $12 \mathrm{~g} / \mathrm{kg} \mathrm{C}$. officinalis Kuan treatment, respectively, compared with SHR with the blood pressure of $187.91 \pm 4.89 \mathrm{mmHg}$. At the 8 th week, there were no differences in the blood pressure between $12 \mathrm{~g} / \mathrm{kg}$ C. officinalis Kuan and enalapril treatment. These results suggest that $C$. officinalis Kuan treatment significantly reduced the blood pressure of SHR.

C. officinalis Kuan treatment inhibits arterial remodeling in $S H R$. It has been suggested previously that an evaluation of arterial alterations may offer valuable information on hypertensive damage of organs in people. In the present study, it evaluated the prevention of $C$. officinalis Kuan from arterial remodeling employing staining with H\&E. As Fig. 1 shows the aorta's medial thickness within the SHR+enalapril, as $\mathrm{SHR}+3 \mathrm{~g} / \mathrm{kg}, \mathrm{SHR}+6 \mathrm{~g} / \mathrm{kg}$ and SHR+12 g/kg, C. officinalis Kuan group was significantly low compared with that of SHR group, with obvious decrease in SHR+6 $\mathrm{g} / \mathrm{kg}$ C. officinalis Kuan group.

C. officinalis Kuan treatment increases NO and eNOS expression and decreases ET-1 and ATIR expression in SHR. As shown in Fig. 2A, the serum level of NO in enalapril and different doses of $C$.officinalis Kuan treatment was significantly increased in comparison to SHR. Measurement of expression shown by ET-1, eNOS and AT1R within aorta used qRT-PCR and western blotting. Different doses of C. officinalis Kuan or enalapril treatment significantly decreased the transcriptional level of ET-1 and AT1R, while increased the transcriptional level of eNOS in the aorta of SHR (Fig. 2B and C). Whereas different doses of $C$. officinalis Kuan treatment significantly reduced the protein level of ET-1 as well as raised eNOS in the aorta of SHR significantly, but had no effect on the protein expression of ATIR (Fig. 2D). Moreover, the transcriptional 

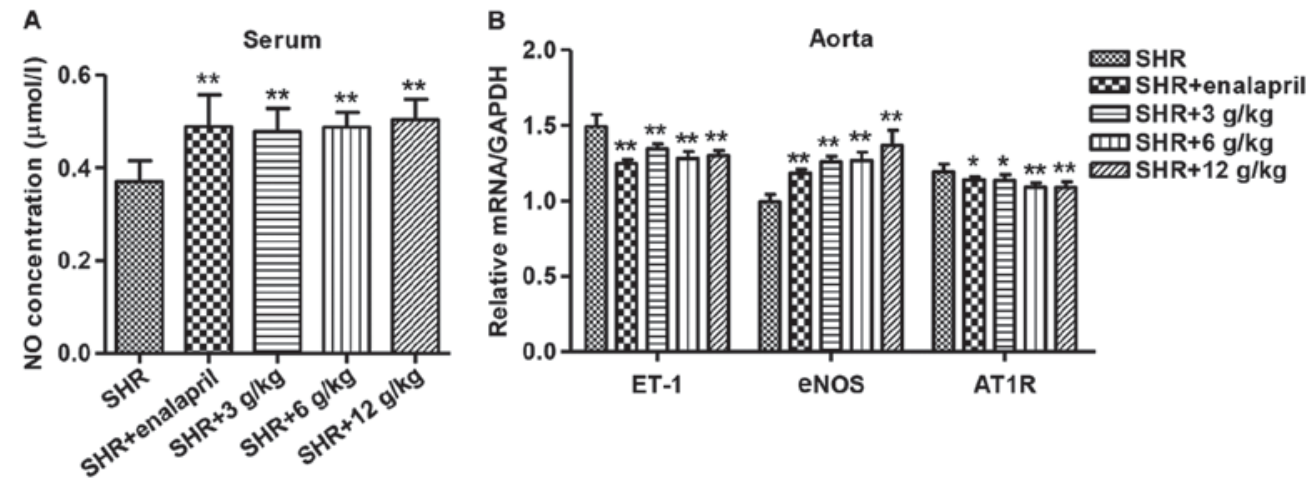

C
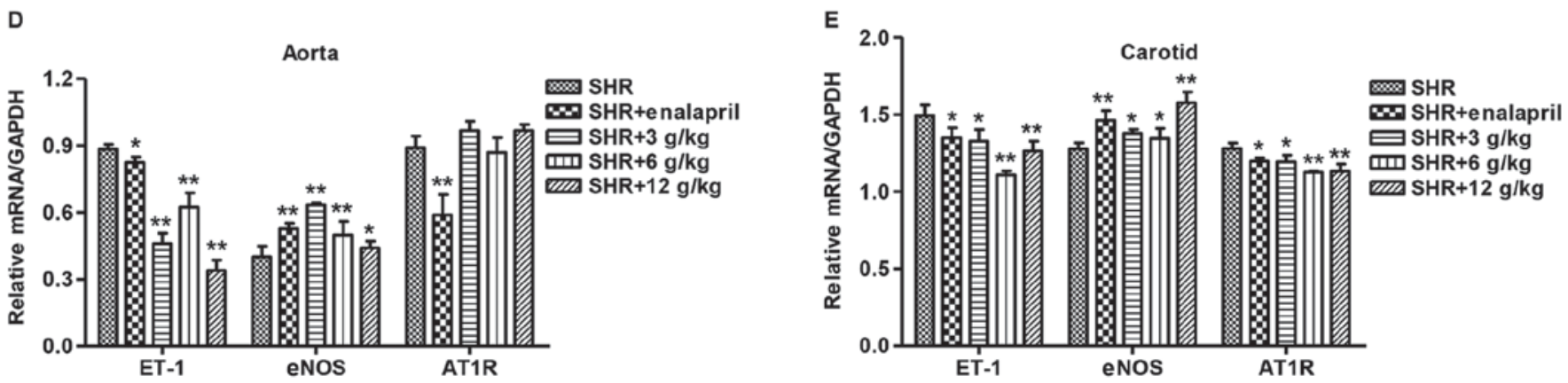

Figure 2. Effect of C. officinalis Kuan on serum NO and expression of ET-1, eNOS and AT1R in SHR. After treatment of SHR with enalapril or different doses of C. officinalis Kuan (3, 6 or $12 \mathrm{~g} / \mathrm{kg}$ ). (A) The serum NO concentration was measured by NO assay kit (nitrate reductase method), (B) the protein and mRNA expression, (C) ET-1, AT1R and eNOS in aorta employed qRT-PCR, and (D) western blotting for measurement and protein in ET-1, eNOS and AT1R in carotid was measured by western blotting. (E) Western blotting for measurement and protein in ET-1, eNOS and AT1R in carotid was measured by western blotting. C. officinalis, Cyathula officinalis; NO, nitric oxide; ET-1, endothelin-1; eNOS, endothelial nitric oxide synthase; AT1R, angiotensin II receptor type 1; SHR, spontaneously hypertensive rat. ${ }^{*} \mathrm{P}<0.05$ and ${ }^{* *} \mathrm{P}<0.01$.

level of ET-1 and AT1R was significantly decreased in the carotid of SHR with enalapril or different doses of $C$. officinalis Kuan treatment, which showed increased transcriptional level of eNOS (Fig. 2E).

\section{Discussion}

Although it was demonstrated in contemporary pharmacological studies that diverse pharmacological activities were possessed by $C$. officinalis Kuan, containing immunostimulant, antitumor, analgesic, anti-inflammatory, eliminating blood stasis, anti-aging, inducing diuresis to treat stranguria, recovering menstrual flow $(15,16)$, it is still unknown whether C. officinalis Kuan would affect arterial change. Within this study, we evaluated the impact of $C$. officinalis Kuan in the procedure of arterial change induced from hypertension and demonstrated that $C$. officinalis Kuan inhibited the blood pressure and arterial ET-1 and AT1R expression as well as increased serum NO level and arterial eNOS expression in SHR. This report is the first to show administration of C. officinalis Kuan improves the arterial remodeling, by decreasing blood pressure, ET-1 and AT1R expression and increasing the $\mathrm{NO}$ and eNOS expression.

Hypertension, a major public health problem, affecting up to one billion people worldwide (17) and exhibiting aortic remodeling including aortic hypertrophy, collagen accumulation and impaired endothelium dependent vasorelaxation (18), among which the main adaptive mechanisms are rearranged formations of extracellular matrix and vascular remodeling to increase blood pressure chronically and growing mortality and morbidity $(19,20)$, characterized in part by the proliferation and hypertrophy of vascular smooth muscle cells (21). Hypertensive vascular remodeling is contributed by the increased vascular cell, inflammation, fibrosis and hypertrophy (22). After C. officinalis Kuan administration for 2 weeks, SHR group had a significant decline in aorta's medial thickness and blood pressure, which was in line with the effect of enalapril in SHR. Enalapril is an orally anti-hypertensive agent with efficacy, affecting risk factors on cardiovascular and preventing decrease within renal function as well as other organ injury positively (23). C. officinalis Kuan presented significant prevention of both vascular function and structure from remodeling, indicating the relation between beneficial impact of $C$. officinalis Kuan and the influence to blood pressure.

In the present study, it was revealed by us that hypertension's pathogenesis is related to NO activity, whereas C. officinalis Kuan may have a hypertensive function that was progressed via elevating the NO level in serum, as well as preventing endothelial impacts, which is in accord with our study that anti-hypertension role is to improve NO production (24). NO belongs to a crucial vasodilator, which is indispensable for maintaining regular blood pressure. Besides, activity of impaired NO takes responsibility for hypertension pathophysiology (25). Hypertension has a pathological feature as the dysfunctional relaxation dependent on endothelium (26). Endothelial vascular cells in the sub-type of $\mathrm{M}$ are activated by $\mathrm{ACh}$, releasing $\mathrm{NO}$, and finally inducing vascular vasodilator (27), resulting in decreased average arterial pressure, sympathetic activity 
and heart rate within rats through activated adenosine $\mathrm{A} 2 \mathrm{~A}$ receptors as well as reduced M1 receptor and ACh levels (28). Accumulating evidence suggests that dysfunctional eNOS, enhanced activity of xanthine oxidase, increased NADPH oxidase activity, and decreased antioxidant defense during the aging process are linked to dysfunction of the endothelium and consequent development of hypertension (29). In the present study, it was discovered that $C$. officinalis Kuan could significantly increase the expression of eNOS in SHR, mimicing the effect of enalapril.

ET-1 affects hypertension. In addition to the impact on people by raising blood pressure, myocardial hypertrophies and vascular are induced by ET-1, as independent risk elements for cardiovascular mortality and morbidity (30). It has been shown that over-activated ET-1 can exacerbate both aortic and cardiac remodeling that could be corrected by ET antagonists $(31,32)$. In the present study, it was discovered that C. officinalis Kuan could significantly decrease the expression of ET-1 and AT1R in SHR, and mimic the effect of enalapril. Enalapril has the ability to decrease plasma levels in Ang II through blocking its last step of activation and offering antihypertensive actions (23). AT1R stimulation regulated ACE2 and Ang-(1-7) expression in aorta of SHR (33). Less activated AT1R as well as their endocellular signaling reduce plasma levels of Ang II (4) and alters the balance of ACE2/Ang-(1-7)/ Mas axis with ACE/Ang II/AT1R axis to improve vascular remodeling (34).

This study was the first to demonstrate that $C$. officinalis Kuan significantly improved arterial remodeling in SHR through decreasing ET-1 and AT1R expression and increasing eNOS and NO expression.

\section{Acknowledgements}

This study was funded by Independent Innovation Research Funding for Putuo District Health System (KW1305), 'Xinglin New Star' Project of Shanghai (ZY3-RCPY-2-2071) and Shanghai Grassroots Senior Experts in Traditional Chinese Medicine Heritage Research Studio Construction Projects (JCZYGZS-020).

\section{References}

1. James PA, Oparil S, Carter BL, Cushman WC, DennisonHimmelfarb C, Handler J, Lackland DT, LeFevre ML, MacKenzie TD, Ogedegbe O, et al: 2014 Evidence-based guideline for the management of high blood pressure in adults: Report from the panel members appointed to the Eighth Joint National Committee (JNC 8). JAMA 311: 507-520, 2014.

2. Derosa G, Bonaventura A, Romano D, Bianchi L, Fogari E, D'Angelo A and Maffioli P: Effects of enalapril/lercanidipine combination on some emerging biomarkers in cardiovascular risk stratification in hypertensive patients. J Clin Pharm Ther 39: 277-285, 2014.

3. Antza C, Stabouli S and Kotsis V: Combination therapy with lercanidipine and enalapril in the management of the hypertensive patient: An update of the evidence. Vasc Health Risk Manag 12: 443-451, 2016.

4. Schiffrin EL: The vascular phenotypes in hypertension: Relation with the natural history of hypertension. J Am Soc Hypertens 1: 56-67, 2007.

5. Lujan HL, Janbaih H and DiCarlo SE: Structural remodeling of the heart and its premotor cardioinhibitory vagal neurons following T(5) spinal cord transection. J Appl Physiol (1985) 116: $1148-1155,2014$
6. Guerrero EI, Ardanaz N, Sevilla MA, Arévalo MA and Montero MJ: Cardiovascular effects of nebivolol in spontaneously hypertensive rats persist after treatment withdrawal. J Hypertens 24: 151-158, 2006.

7. Gómez-Roso M, Montero MJ, Carrón R and Sevilla MA: Cardiovascular changes in spontaneously hypertensive rats are improved by chronic treatment with zofenopril. Br J Pharmacol 158: 1911-1921, 2009.

8. Schiffrin EL: Vascular remodeling in hypertension: Mechanisms and treatment. Hypertension 59: 367-374, 2012.

9. Higashi Y, Sasaki S, Nakagawa K, Ueda T, Yoshimizu A, Kurisu S, Matsuura H, Kajiyama G and Oshima T: A comparison of angiotensin-converting enzyme inhibitors, calcium antagonists, beta-blockers and diuretic agents on reactive hyperemia in patients with essential hypertension: A multicenter study. J Am Coll Cardiol 35: 284-291, 2000.

10. Schmidt BM, Ribnicky DM, Lipsky PE and Raskin I: Revisiting the ancient concept of botanical therapeutics. Nat Chem Biol 3: 360-366, 2007.

11. Zhou R, Li BG and Zhang GL: Chemical study on Cyathula officinalis Kuan. J Asian Nat Prod Res 7: 245-252, 2005.

12. Feng H, Du X, Liu J, Han X, Cao X and Zeng X: Novel polysaccharide from Radix Cyathulae officinalis Kuan can improve immune response to ovalbumin in mice. Int J Biol Macromol 65: 121-128, 2014.

13. Park HY, Lim H, Kim HP and Kwon YS: Downregulation of matrix metalloproteinase-13 by the root extract of Cyathula officinalis Kuan and its constituents in IL-1 $\beta$-treated chondrocytes. Planta Med 77: 1528-1530, 2011.

14. Liu J, Xu J, Zhao XJ, Gao WY, Zhang SZ and Guo YQ: A new heterocyclic compound from Cyathula officinalis Kuan. Chin Chem Lett 21: 70-72, 2010.

15. Ye P, Peng J and Liu J: The research development of Cyathula officinalis kuan. Zhongguo Yaowu Huaxue Zazhi 35: 51-53, 2007.

16. Han X, Shen S, Liu T, Du X, Cao X, Feng H and Zeng X: Characterization and antioxidant activities of the polysaccharides from Radix Cyathulae officinalis Kuan. Int J Biol Macromol 72: 544-552, 2015 .

17. Kearney PM, Whelton M, Reynolds K, Muntner P, Whelton PK and He J: Global burden of hypertension: Analysis of worldwide data. Lancet 365: 217-223, 2005.

18. Yu TT, Guo K, Chen HC, Lan CZ, Wang J, Huang LL, Wang XH, Zhang Z and Gao S: Effects of traditional Chinese medicine Xin-Ji-Er-Kang formula on 2K1C hypertensive rats: Role of oxidative stress and endothelial dysfunction. BMC Complement Altern Med 13: 173, 2013.

19. Ghiadoni L, Taddei S and Virdis A: Hypertension and endothelial dysfunction: Therapeutic approach. Curr Vasc Pharmacol 10: 42-60, 2012.

20. Shantsila A and Shantsila E: Arterial stiffening in hypertension: Beyond blood pressure levels. J Hum Hypertens 24: 303-305, 2010.

21. Feihl F, Liaudet L, Levy BI and Waeber B: Hypertension and microvascular remodelling. Cardiovasc Res 78: 274-285, 2008.

22. Gao P, Xu TT, Lu J, Li L, Xu J, Hao DL, Chen HZ and Liu DP: Overexpression of SIRT1 in vascular smooth muscle cells attenuates angiotensin II-induced vascular remodeling and hypertension in mice. J Mol Med (Berl) 92: 347-357, 2014.

23. Barrios V, Escobar C and Echarri R: Fixed combinations in the management of hypertension: Perspectives on lercanidipine-enalapril. Vasc Health Risk Manag 4: 847-853, 2008.

24. Jiang H, Shen Z, Chu Y, Li Y, Li J, Wang X, Yang W, Zhang X, $\mathrm{Ju} \mathrm{J}, \mathrm{Xu} \mathrm{J}$, et al: Serum metabolomics research of the anti-hypertensive effects of Tengfu Jiangya tablet on spontaneously hypertensive rats. J Chromatogr B Analyt Technol Biomed Life Sci 1002: 210-217, 2015.

25. Gordish KL, Kassem KM, Ortiz PA and Beierwaltes WH: Moderate (20\%) fructose-enriched diet stimulates salt-sensitive hypertension with increased salt retention and decreased renal nitric oxide. Physiol Rep 5: 5, 2017.

26. Zhai YL, Zhu L, Shi SF, Liu LJ, Lv JC and Zhang H: Elevated soluble VEGF receptor sFlt-1 correlates with endothelial injury in IgA nephropathy. PLoS One 9: e101779, 2014.

27. Wassmann S, Laufs U, Stamenkovic D, Linz W, Stasch JP, Ahlbory K, Rösen R, Böhm M and Nickenig G: Raloxifene improves endothelial dysfunction in hypertension by reduced oxidative stress and enhanced nitric oxide production. Circulation 105: 2083-2091, 2002. 
28. Jiang MY, Chen J, Wang J, Xiao F, Zhang HH, Zhang CR, Du DS, Cao YX, Shen LL and Zhu DN: Nitric oxide modulates cardiovascular function in the rat by activating adenosine $\mathrm{A} 2 \mathrm{~A}$ receptors and inhibiting acetylcholine release in the rostral ventrolateral medulla. Clin Exp Pharmacol Physiol 38: 380-386, 2011.

29. Yang YM, Huang A, Kaley G and Sun D: eNOS uncoupling and endothelial dysfunction in aged vessels. Am J Physiol Heart Circ Physiol 297: H1829-H1836, 2009.

30. Zhang X, Wang X, Hu F, Zhou B, Chen HB, Zha D, Liu Y, Guo Y, Zheng L and Xiu J: A novel hydrodynamic approach of drag-reducing polymers to improve left ventricular hypertrophy and aortic remodeling in spontaneously hypertensive rats. Int J Nanomed 11: 6743-6751, 2016.

31. Lee TM, Lin MS, Chou TF, Tsai CH and Chang NC: Effect of pravastatin on development of left ventricular hypertrophy in spontaneously hypertensive rats. Am J Physiol Heart Circ Physiol 289: H220-H227, 2005.
32. Amiri F, Virdis A, Neves MF, Iglarz M, Seidah NG, Touyz RM, Reudelhuber TL and Schiffrin EL: Endothelium-restricted overexpression of human endothelin-1 causes vascular remodeling and endothelial dysfunction. Circulation 110: 2233-2240, 2004.

33. Igase M, Strawn WB, Gallagher PE, Geary RL and Ferrario CM: Angiotensin II AT1 receptors regulate ACE2 and angiotensin-(1-7) expression in the aorta of spontaneously hypertensive rats. Am J Physiol Heart Circ Physiol 289: H1013-H1019, 2005.

34. Iwai M, Nakaoka H, Senba I, Kanno H, Moritani T and Horiuchi M: Possible involvement of angiotensin-converting enzyme 2 and Mas activation in inhibitory effects of angiotensin II type 1 receptor blockade on vascular remodeling. Hypertension 60: 137-144, 2012. 